\title{
OPTICAL CONDUCTIVITY OF METAL NANOSHELLS
}

\author{
P. M. Tomchuk ${ }^{1}$, V. V. Kulish ${ }^{2}$ \\ ${ }^{1}$ Institute of Physics, Department of Theoretical Physics, \\ 46 Nauki prosp., Kyiv, UA-03028, Ukraine \\ ${ }^{2}$ Physical-Technical Institute, NTUU "KPI", Department of Applied Physics, \\ 37 Peremogy prosp., Kyiv, UA-03056, Ukraine \\ e-mail: kulish_volv@ukr.net
}

(Received April 9 2003; received in final form October 1, 2003)

\begin{abstract}
In this work the expression for optical conductivity of spherical metal nanoshell as a function of internal and external radii of nanoshell and photon energy - Fermi energy ratio is obtained. Quantization of electron energy in nanoshells is shown to lead to the appearance of an oscillating dependence of optical conductivity on the light frequency. An explicit expression of oscillating addends for optical conductivity is obtained.
\end{abstract}

Key words: nanoshells, small clusters, optical conductivity, oscillations, spectrum quantization.

PACS number(s): 36.40.Vz, 61.46.+w, 73.50.Pz, 78.20.Jq, 78.66.Bz, 78.67.Bf

\section{INTRODUCTION}

In recent years the research of physical properties of various nanostructures has become significant and popular. It is known that physical properties of small particles and their ensembles differ drastically from similar properties of bulk substance. In particular, when we deal with ensembles of metal particles, new phenomena like electron and photon emission at the introducion of power into ensemble [1] appear. These phenomena happen at the power that is relatively small so that in bulk metal they are not observed. A multitude of new effects is observed in the absorption of light by small metal particles (in particular, a steep dependence of absorption from the shape of a particle and polarization of electromagnetic wave appear [2]).

In this work, optical conductivity of metal nanoshells is studied theoretically. An experimental study of such systems is described in [3]. Our task is to find out how effect of quantization of electron spectrum in spherical metal nanoshells affects optical conductivity.

\section{SETTING OF THE PROBLEM}

Let's study spherical metal nanoshell with the internal radius $r=a$ and external radius $r=b$. The potential energy of an electron in such a shell as a function of the radial coordinate $V(r)$ is given on Fig. 1.

We investigate light absorption in metal nanoshell. First, we should find eigenfunctions and energy spectrum of the electron in the potential well $V(r)$. The Hamiltonian of our system can be written as follows:

$$
\hat{H}=\frac{\hat{\mathbf{p}}^{2}}{2 m_{0}}+V(r)
$$

Here, $\hat{\mathbf{p}}$ is the momentum operator of the electron, $m_{0}$ is its effective mass.
The eigenfunctions of the Hamiltonian $\hat{H}$ can be represented in the form

$$
\psi(\mathbf{r})=R(r) Y_{l m}(\theta, \varphi)
$$

Here $Y_{l m}(\theta, \varphi)$ are spherical functions, the radial function $R(r)$ must satisfy the following equation:

$R^{\prime \prime}+\frac{2}{r} R^{\prime}+\left(\frac{2 m_{0} E}{\hbar^{2}}-\frac{l(l-1)}{r^{2}}\right) R=0, \quad r \in(a, b)$

Here $E$ is the electron energy, $l$ is the orbital quantum number.

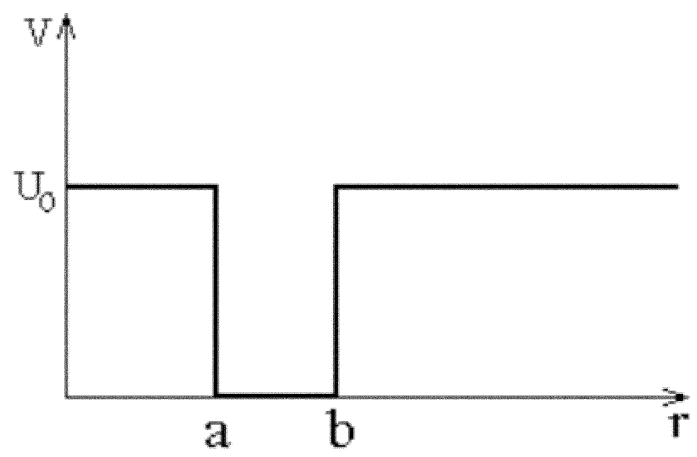

Fig. 1. The potential in which the electron moves as a function of radial coordinate for a model potential of the nanoshell. $U_{0}$ is the dielectric barrier height, which is supposed to be equal outside the particle and in the inner cavity, $a$ and $b$ are internal and external radii of nanoshell, correspondingly.

The solution of (3) can be written in the form

$$
R(r)=B_{1} j_{l}(k r)+B_{2} n_{l}(k r)
$$


where $j_{l}$ and $n_{l}$ are the spherical Bessel functions, $B_{1}$ and $B_{2}$ are the constants.

Also, we should mention that

$$
\hbar k=\sqrt{2 m_{0} E}
$$

To equation (3) we must add boundary conditions. If weak lowering of wavefunction in the barrier is neglected, boundary conditions can be written as follows:

$$
R(a)=R(b)=0
$$

( $a$ and $b$ are internal and external radii of nanoshell, correspondingly). Condition (6) is satisfied exactly for a barrier with infinite height.

Then, we presume that condition

$$
k a \geq 1
$$

is satisfied.

From (5) we can see that this condition is satisfied, for instance, if electron energy has the order of magnitude of Fermi energy and $a \geq 2 \cdot 10^{-7} \mathrm{~cm}$.

From (7) we can see that condition $k r \gg 1$ is satisfied for $r \in(a, b]$ and for the vicinity of $a$ and $b$; we use this in our further calculations.

If (7) is satisfied, we can use asymptotics for the functions $j_{l}$ and $n_{l}$ :

$$
\begin{aligned}
& j_{l}(k r) \approx \frac{1}{k r} \cos \left(k r-(l+1) \frac{\pi}{2}\right), \\
& n_{l}(k r) \approx \frac{1}{k r} \sin \left(k r-(l+1) \frac{\pi}{2}\right) .
\end{aligned}
$$

Then, we introduce amplitude and phase instead of constants $B_{1}$ and $B_{2}$ :

$$
B_{1}=B \sin \alpha, \quad B_{2}=B \cos \alpha
$$

so the asymptotics of normalized on 1 function (4) can be written as

$$
R(r) \approx\left(\frac{2}{b-a}\right)^{\frac{1}{2}} \frac{1}{r} \sin \left(k r-(l+1) \frac{\pi}{2}+\alpha\right)
$$

Let's denote the values of $k$ that satisfy (6) as $k_{n l}$. Taking into consideration (10), we can see that conditions (6) are satisfied if

$$
\begin{aligned}
& k_{n l} a-(l+1) \pi / 2+\alpha_{n l}=\pi n, \\
& k_{n l}(b-a)=\pi n .
\end{aligned}
$$

The second condition in (11) means that the interval between $a$ and $b$ contains an integer number of wavelength- es.

Replacing $k$ with $k_{n l}$ in (5), we obtain quantum levels of electron energy

$$
E=E_{n l}=\frac{\hbar^{2}}{2 m_{0}}\left(\frac{\pi n}{b-a}\right)^{2}
$$

As we can see, energy is degenerated by orbital and azimuthal quantum numbers.

To be strict, this claim needs to be elaborated. More precise calculations (see Appendix) give instead of (12)

$$
E_{n l}=\frac{\hbar^{2}}{2 m_{0}}\left(\left(\frac{\pi n}{b-a}\right)^{2}+\frac{l(l+1)+1}{b^{2}}\right)
$$

so $E_{n l}$ is a function of $l$. But taking into consideration the fact that $b \gg b-a$ and $l \leq 2 n$, we can see that the second addend in brackets is negligible as compared with the first one. After neglecting this addend, we obtain (12). Even for $b \sim b-a$ (a small value of $a$ ) and $l=2 n$ the second addend is by 2.5 times less than the first one.

Now let us take into consideration the finiteness of a potential well. We suppose that the difference of a disturbed wavefunction $V_{0}<+\infty, E<V_{0}, K_{n l} a \gg 1$, where $K_{n l}=\sqrt{2 m\left(V_{0}-E\right)} / \hbar \approx \sqrt{2 m V_{0}} \hbar$ from the nondisturbed one can be described with small addends to $k$ and $\alpha$ :

$R_{n l}(r)=\frac{\tilde{B}}{r} \sin \left(\left(k_{n l}+\Delta k_{n l}\right) r-\frac{\pi}{2}(l+1)+(\alpha+\Delta \alpha)\right)$,

$r \in(a, b]$

It can be easily seen that for $r \notin(a, b]$

$$
\begin{aligned}
& r \leq a, \quad K_{n l} r \gg 1, \\
& R_{n l}=\tilde{A} j_{l}\left(i K_{n l} r\right) \approx \frac{A}{r} \exp \left(K_{n l} r\right)
\end{aligned}
$$

in an analogous way

$$
r>b, \quad R_{n l} \approx \frac{C}{r} \exp \left(-K_{n l} r\right)
$$

(here $\tilde{A}, A$ and $C$ are the normalization constants) we will find small addends to $k$ and $\alpha$ from the condition of sewing the radial part of the wavefunction for $r=a$ and $r=b$, we use sewing of a logarithmic derivative:

$$
r \rightarrow b+0: \quad \frac{R_{n l}^{\prime}(b+0)}{R_{n l}(b+0)} \approx-K_{n l}, \quad \text { as } \quad K_{n l} b \gg 1,
$$




$$
r \rightarrow a-0: \quad \frac{R_{n l}^{\prime}(a-0)}{R_{n l}(a-0)} \approx K_{n l}, \quad \text { as } \quad K_{n l} a \gg 1 .
$$

Taking into consideration only addends of the first order of magnitude, we can easily obtain

$$
\begin{aligned}
& \frac{R_{n l}^{\prime}(b-0)}{R_{n l}(b-0)}=\frac{k_{n l}}{\Delta k_{n l} b+\Delta \alpha}, \\
& \frac{R_{n l}^{\prime}(a+0)}{R_{n l}(a+0)}=\frac{k_{n l}}{\Delta k_{n l} a+\Delta \alpha} .
\end{aligned}
$$

From the condition of sewing the logarithmic derivative we obtain

$$
\left\{\begin{array}{l}
\Delta k_{n l} b+\Delta \alpha=-k_{n l} / K_{n l} \\
\Delta k_{n l} a+\Delta \alpha=k_{n l} / K_{n l}
\end{array}\right.
$$

(We do not calculate $\Delta k_{n l}$ and $\Delta \alpha$ because for further calculations we need not more exact expressions of the electronic spectrum and phase but the value of wavefunction on the border of the potential well; it is obtained from the combinations of $\Delta k_{n l}$ and $\Delta \alpha$ given above).

We have obtained the wavefunctions and energy spectrum of the electron in a metal shell. Now we start the study of light absorption.

\section{LIGHT ABSORPTION}

The Hamiltonian of the electron interaction with an electromagnetic wave field can be written in the form

$$
\hat{H}_{\mathrm{int}}=-\frac{e}{m_{0} c} \mathbf{A} \hat{\mathbf{p}}
$$

Here $e$ is electron charge, $c$ is light velocity, $\mathbf{A}$ is vectorpotential of the electromagnetic field.

We suppose that $\mathbf{A}$ can be written in the form

$$
\mathbf{A}=\mathbf{A}_{0} \sin \omega t
$$

where $\omega$ is light frequency.

Let us direct the $z$ axis along $\mathbf{A}_{0}$. Then we can write $\hat{H}_{\text {int }}$ in the form

$$
\hat{H}_{\mathrm{int}}=i \frac{e A_{0}}{2 m_{0} c} \hat{p}_{z}\left(e^{i \omega t}-e^{-i \omega t}\right) \equiv \hat{H}^{\prime} \cdot\left(e^{i \omega t}-e^{-i \omega t}\right) .
$$

The energy that is absorbed by the electron system during a unit of time is given by the following expression:

$$
\begin{aligned}
W & =\frac{2 \pi}{\hbar} \sum_{(i, f)}\left|\left\langle i\left|\hat{H}^{\prime}\right| f\right\rangle\right|^{2}\left(E_{i}-E_{f}\right) f\left(E_{i}\right)\left(1-f\left(E_{f}\right)\right) \\
& \times \delta\left(E_{f}-E_{i}-\hbar \omega\right)
\end{aligned}
$$

In (21) index $i$ means the initial and $f$, respectively, the final state, $f(E)$ us a function of electrons distribution by energy.

For the sake of simplicity we denote the energy of the initial state $E_{i}$ as $E$ and that of the final state $E_{f}$ as $E^{\prime}$.

In correspondence with (20) we can write

$$
\left\langle i\left|\hat{H}^{\prime}\right| f\right\rangle=i \frac{e A_{0}}{2 m_{0} c}\left\langle i\left|\hat{p}_{z}\right| f\right\rangle \equiv-\frac{i e E_{0}}{2 m_{0} c}\left\langle i\left|\hat{p}_{z}\right| f\right\rangle .
$$

In this equation we expressed the amplitude of the vector-potential $A_{0}$ through amplitude of the electrical field of the wave $\left(E_{0}=-\omega A_{0} / c\right)$.

The matrix element of momentum operator $\hat{p}$ can be easily calculated using functions (2) and (10):

$$
\left\langle i\left|\hat{p}_{z}\right| f\right\rangle \equiv \int d \mathbf{r} R_{n l}(r) Y_{l m}(\theta, \varphi) \hat{p}_{z} R_{n^{\prime} l^{\prime}}(r) Y_{l^{\prime} m^{\prime}}(\theta, \varphi) .
$$

The function $R_{n l}$ in (23) is obtained from (10) after the replacement of $k$ and $\alpha$ with $k_{n l}$ and $\alpha_{n l}$; also, $\langle i| \equiv\langle n l m|$ and $|f\rangle \equiv\left|n^{\prime} l^{\prime} m^{\prime}\right\rangle$.

The integral by $r$ in (23) can be easily calculated, we obtain

$$
\begin{aligned}
\left\langle i\left|\hat{p}_{z}\right| f\right\rangle & =\frac{2 i \hbar}{b-a} \frac{k_{n l} k_{n^{\prime} l^{\prime}}}{k_{n l}^{2}-k_{n^{\prime} l^{\prime}}^{2}}\left(1-(-1)^{n+n^{\prime}}\right) \\
& \times \int d \Omega Y_{l m}(\theta, \varphi) Y_{l^{\prime} m^{\prime}}^{*}(\theta, \varphi) \cos \theta .
\end{aligned}
$$

An integral by the angle can also be easy calculated:

$$
\int d \Omega Y_{l m}(\theta, \varphi) Y_{l^{\prime} m^{\prime}}^{*}(\theta, \varphi) \cos \theta=\left(\left(\frac{\left(l^{\prime}+m^{\prime}\right)\left(l^{\prime}-m^{\prime}\right)}{\left(2 l^{\prime}+1\right)\left(2 l^{\prime}-1\right)}\right)^{\frac{1}{2}} \delta_{l, l^{\prime}-1}+\left(\frac{(l+m)(l-m)}{(2 l+1)(2 l-1)}\right)^{\frac{1}{2}} \delta_{l, l^{\prime}+1}\right) \delta_{m, m^{\prime}} .
$$

From (11) we can see that

$$
k_{n l}=\frac{\pi}{b-a} n \equiv k(n)
$$


so $k_{n l}$ is not a function of $l$. It means that after substituting $\left|\left\langle i\left|\hat{H}^{\prime}\right| f\right\rangle\right|^{2}$ into (21) we can perform summation over $(l, m)$ and over $\left(l^{\prime}, m^{\prime}\right)$. Summation over $m$ is performed in limits $-l \leq m \leq l$ and $l$ changes from 0 to $2 n, l^{\prime}$ and $m^{\prime}$ change correspondingly. But the presence of delta-indices $\delta_{m, m^{\prime}}$ and $\delta_{l, l^{\prime}+1}$ changes the picture of summation. In fact, $l$ and $l^{\prime}$ change from 0 to $2 \min \left(n, n^{\prime}\right) \pm 1$. If absorption take place, $n$ is less than $n^{\prime}$, so $0 \leq l, l^{\prime} \leq 2 n$ (we neglect unity comparing to $2 n$ ). Having made this notations, we can write

$$
W=\frac{4 \pi}{3} \frac{\left(e E_{0}\right)^{2} \cdot \hbar^{4}}{m_{0}^{4} \omega^{3}(b-a)^{2}} \sum_{n, n^{\prime}}\left(1-(-1)^{n-n^{\prime}}\right) k^{2}(n) k^{2}\left(n^{\prime}\right) n^{2} f(E)\left(1-f\left(E^{\prime}\right)\right) \delta\left(E^{\prime}-E-\hbar \omega\right) .
$$

We remind that $E$ is given by (12) and $E^{\prime}$ can be obtained from (12) after replacing $n$ with $n^{\prime}$.

The power absorbed by electronic system can also be expressed through optical conductivity $\sigma(\omega)$ in the following way:

$$
W=\frac{1}{2} V_{b} \sigma(\omega) E_{0}^{2}
$$

Here $\mathrm{V}_{b}$ is the volume of metal shell. Comparing this with (27), we obtain the expression for $\sigma(\omega)$

$$
W=\frac{8 \pi}{3 V_{b}} \frac{e^{2} \hbar^{4}}{m_{0}^{4} \omega^{3}(b-a)^{2}} \sum_{n, n^{\prime}}\left(1-(-1)^{n-n^{\prime}}\right) n^{2} k^{2}(n) k^{2}\left(n^{\prime}\right) f(E)\left(1-f\left(E^{\prime}\right)\right) \delta\left(E^{\prime}-E-\hbar \omega\right)
$$

In this paragraph we neglect discreteness of electronic spectrum and replace in (28) the sum with integral. At this we take into account that

$$
\Delta n=\frac{b-a}{\pi} \Delta k, \quad \Delta n^{\prime}=\frac{b-a}{\pi} \Delta k^{\prime}
$$

The function $\left(1-(-1)^{n-n^{\prime}}\right)$ takes discrete values $0,2,0,2, \ldots$, so we replace it with its mean, i. e., unity. So we obtain

$$
\sigma_{0}(\omega)=\frac{8 e^{2}(b-a)^{2} \hbar^{4}}{3 \pi^{3} m_{0}^{4} \omega^{3} V_{b}} \int_{0}^{\infty} \int_{0}^{\infty} d k d k^{\prime} k^{4}{k^{\prime}}^{2} f(E)\left(1-f\left(E^{\prime}\right)\right) \delta\left(E^{\prime}-E-\hbar \omega\right)
$$

The integral by $k$ and $k^{\prime}$ can easily be calculated after following an approximation of the function $f(E)$

$$
f(E)=1-\chi\left(E-E_{f}\right),
$$

where $\chi$ is the Heaviside function and $E_{f}$ is Fermi energy. First, we make substitution

$$
k^{2}=\frac{2 m_{0}}{\hbar^{2}} E, \quad k d k=\frac{m_{0}}{\hbar^{2}} d E .
$$

We obtain

$$
\begin{aligned}
& \int_{0}^{+\infty} \int_{0}^{+\infty} k^{4}\left(k^{\prime}\right)^{2} f(E)\left(1-f\left(E^{\prime}\right)\right) \delta\left(E-E^{\prime}-\hbar \omega\right) d k d k^{\prime} \\
& =\int_{0}^{+\infty} \int_{0}^{+\infty}\left(\frac{2 m_{0}}{\hbar^{2}} E\right)^{\frac{3}{2}}\left(\frac{m_{0}}{\hbar^{2}} E^{\prime}\right)^{\frac{1}{2}}\left(\frac{m_{0}}{\hbar^{2}}\right) f(E)\left(1-f\left(E^{\prime}\right)\right) \delta\left(E-E^{\prime}-\hbar \omega\right) d E d E^{\prime}
\end{aligned}
$$

(after using (31)) 


$$
\approx\left(\frac{m_{0}}{\hbar^{2}}\right)^{2}\left(\frac{2 m_{0}}{\hbar^{2}}\right)^{2} \int_{E_{f}-\hbar \omega}^{E_{f}} E^{\frac{3}{2}} \sqrt{E+\hbar \omega} d E=\left(\frac{m_{0}}{\hbar^{2}}\right)^{2}\left(\frac{2 m_{0}}{\hbar^{2}}\right)^{2} E_{f}^{3} \int_{1-\nu}^{1} q^{\frac{3}{2}} \sqrt{q+\nu} d q=\left(\frac{m_{0}}{\hbar^{2}}\right)^{2}\left(\frac{2 m_{0}}{\hbar^{2}}\right)^{2} E_{f}^{3} \tilde{f}(\nu),
$$

where $\nu=\hbar \omega / E_{f}$ and

$$
\tilde{f}(\nu)=\int_{1-\nu}^{1} q^{3 / 2} \sqrt{q+\nu} d q=\left.\left(\frac{(q(q+\nu))^{3 / 2}}{3}-\frac{\nu(2 q+\nu) \sqrt{q(q+\nu)}}{8}+\frac{\nu^{3}}{8} \ln (\sqrt{q}+\sqrt{q+\nu})\right)\right|_{1-\nu} ^{1} .
$$

For optical conductivity

$$
\sigma_{1}=\frac{8 e^{2} \hbar^{4}(b-a)^{2}}{3 \pi^{3} m_{0}^{4} \omega^{3} V_{b}}\left(\frac{m_{0}}{\hbar^{2}}\right)^{2}\left(\frac{2 m_{0}}{\hbar^{2}}\right)^{2} E_{f}^{3} \tilde{f}(\nu)=\frac{32 e^{2}(b-a)^{2}}{3 \pi^{3} \hbar^{4} \omega^{3} V_{b}} E_{f}^{3} \tilde{f}(\nu),
$$

and as nanoshell volume

$$
V_{b}=\frac{4 \pi}{3}\left(b^{3}-a^{3}\right)
$$

we can write

$$
\sigma_{1}=\frac{32 e^{2}(b-a)^{2}}{3 \pi^{3} \hbar^{4} \omega^{3}} \frac{3}{4 \pi\left(b^{3}-a^{3}\right)} E_{f}^{3} \tilde{f}(\nu)=\frac{8 e^{2}(b-a)^{2}}{\pi^{4} \hbar\left(b^{3}-a^{3}\right)} \frac{E_{f}^{3}}{(\hbar \omega)^{3}} \tilde{f}(\nu)=\frac{8 e^{2}(b-a)^{2}}{\pi^{4} \hbar\left(b^{3}-a^{3}\right)} f(\nu),
$$

where

$$
f(\nu)=\frac{\tilde{f}(\nu)}{\nu^{3}}
$$

so sought optical conductivity

$$
\sigma_{1}=\frac{8 e^{2}}{\pi^{4} \hbar} \frac{(b-a)^{2}}{b^{3}-a^{3}} f(\nu)
$$

where $\nu=\hbar \omega / E_{f}$ and function $\mathrm{f}(\nu)$ is given by the following expression:

$$
f(\nu)=\nu^{-3} \int_{1-\nu}^{1} q^{3 / 2} \sqrt{q+\nu} d q=\left.\frac{1}{\nu^{3}}\left(\frac{(q(q+\nu))^{3 / 2}}{3}-\frac{\nu(2 q+\nu) \sqrt{q(q+\nu)}}{8}+\frac{\nu^{3}}{8} \ln (\sqrt{q}+\sqrt{q+\nu})\right)\right|_{1-\nu} ^{1} .
$$

For the case $\nu \equiv \hbar \omega / E_{f} \ll 1(33)$ gives

$$
f(\nu) \approx \nu^{-2},
$$

so in this limit dependence optical conductivity from frequency is the same as for bulk metal.

\section{OSCILLATIONS OF OPTICAL CONDUCTIVITY AS A RESULT OF QUANTITIZATION OF THE ELECTRONIC SPECTRUM}

In this paragraph we shall investigate the displays of discrete nature of electronic spectrum in optical conductivity (and, consequently, in absorption). Let us rewrite equation (28) in the following form: 


$$
\begin{aligned}
W & =\frac{8 \pi}{3 V_{b}} \frac{e^{2} \hbar^{4}}{m_{0}^{4} \omega^{3}(b-a)^{2}} \sum_{n} n^{2}\left(k^{2}(n)+\frac{2 m_{0} \omega}{\hbar}\right) k^{2}(n) f(E)(1-f(E+\hbar \omega)) \\
& \times \sum_{n^{\prime}}\left(1-(-1)^{n^{\prime}-n}\right) \delta\left(E^{\prime}-E-\hbar \omega\right) .
\end{aligned}
$$

In (35) we used energy conservation law and expressed $E^{\prime}$ through $E$ and $k^{2}\left(n^{\prime}\right)$ through $k^{2}(n)$. Let us remind that dependence of $E$ on $n$ is given by (12), $E^{\prime}$ depends on $n^{\prime}$ in the same way.

Instead of replacing the sum with integral here we use the exact formula for summation - the Poisson formula:

$$
\sum_{n=1}^{\infty} y(n)=\int_{0}^{\infty} d n\left(y(n)+2 \sum_{s=1}^{\infty} y(n) \cos (2 \pi s n)\right)
$$

Such a procedure is used in theory of de Gaaz-van Alfen oscillations (see, for instance, [4]).

Applying the Poisson formula for summation over $n$ does not make any difficulties. But when we use this formula for summation over $n^{\prime}$ we need to take into account some specific points. We should calculate the following sum:

$$
\begin{aligned}
G(E(n)+\hbar \omega) & \equiv \sum_{n^{\prime}=1}^{\infty}\left(1-(-1)^{n-n^{\prime}}\right) \\
& \times \delta\left(E\left(n^{\prime}\right)-E(n)-\hbar \omega\right)
\end{aligned}
$$

The first notation is the following: $\delta$-function of discrete indices ( $n$ and $n^{\prime}$ ) has no mathematical sense. If we want to use it, we should remember that the $\delta$-function in physics is, in fact, a limit of some classical function (for example, from $C^{\infty}$ or given below). After taking the limit area under the graph of classical function remains equal to 1 when the width of the figure under the graph tends to zero and its height to infinity. We use the following classical function:

$$
\delta^{*}(x)=\left\{\begin{array}{lr}
0, & x<-\Delta E / 2 \\
1 / \Delta E, & -\Delta E / 2<x<\Delta E / 2 \\
0, & x>\Delta E / 2
\end{array}\right.
$$

with $\Delta E \rightarrow 0$ limit.

The second note concerns the factor $\left(1-(-1)^{n^{\prime}-n}\right)$ that equals 0 or 2 depending on whether from the fact, is the number $n^{\prime}-n$ even or odd. In the previous paragraph we replaced this function with its mean (unity). If we take into consideration discreteness of spectrum, the possibility of such an approximation should be proved. It can be proved if we consider for (37) two situations ( $n$ being even and $n$ being odd) separately. For example, if $n$ in (37) is odd, only even $n\left(n^{\prime}=2 m^{\prime}\right.$, where $\left.m^{\prime}=1,2,3, \ldots\right)$ will remain in the sum. Then,

$$
G(E(n)+\hbar \omega)=2 \sum_{m^{\prime}=1}^{\infty} \delta^{*}\left(E\left(2 m^{\prime}\right)-E(n)-\hbar \omega\right)
$$

Now we use (36) to calculate the sum (39):

$$
\begin{aligned}
G(E(n)+\hbar \omega) & =\int_{0}^{\infty} d m^{\prime} \cdot 2 \delta^{*}\left(E\left(2 m^{\prime}\right)-E(n)-\hbar \omega\right)\left(1+2 \sum_{s=1}^{\infty} \cos \left(2 \pi s \cdot 2 m^{\prime}\right)\right) \\
& =\int_{0}^{\infty} d n^{\prime} \delta^{*}\left(E\left(n^{\prime}\right)-E(n)-\hbar \omega\right)\left(1+2 \sum_{s=1}^{\infty} \cos \left(2 \pi s n^{\prime}\right)\right) .
\end{aligned}
$$

We can see that the result is the same as if we replaced $\left(1-(-1)^{n-n^{\prime}}\right)$ with 1 from the beginning. For even $n$ this fact could be proved in the same way (in this case only odd $n^{\prime}\left(n^{\prime}=2 m^{\prime}-1\right)$ makes an essential contribution).

So we replace in $(37)$ the discrete function $\left(1-(-1)^{n-n^{\prime}}\right)$ with its mean that equals one and the delta-function with $\delta^{*}$ and use the Poisson formula for a resulting sum. We obtain

$$
G(E+\hbar \omega)=\frac{(b-a) \sqrt{2 m_{0}}}{2 \pi \hbar \sqrt{E+\hbar \omega}}\left(1+2 \sum_{s=1}^{\infty} \cos \left(s \frac{2(b-a)}{\hbar} \sqrt{2 m_{0}(E+\hbar \omega)}\right)\right)
$$

For the sake of simplicity we throw away index $n$ for $E_{n}$ in (40). Also, in (40) we have already made limit transition 
$\Delta E \rightarrow 0$ (here $\Delta E$ is the same as in (38)). After substituting $G(E+\hbar \omega)$ into (35) we use one more time (36) for the summation over $n$. We obtain

$$
\begin{aligned}
\sigma_{0}(\omega) & =\frac{32 e^{2}(b-a)^{2}}{3(\pi \hbar \omega)^{3} \hbar V_{b}} \int_{0}^{\infty} d E \cdot E^{\frac{3}{2}}(E+\hbar \omega)^{\frac{1}{2}} f(E)(1-f(E+\hbar \omega)) \\
& \times\left(1+2 \sum_{s=1}^{\infty} \cos \left(s \frac{2(b-a)}{\hbar} \sqrt{2 m_{0} E}\right)\right)\left(1+2 \sum_{s^{\prime}=1}^{\infty} \cos \left(s^{\prime} \frac{2(b-a)}{\hbar} \sqrt{2 m_{0}(E+\hbar \omega)}\right)\right)
\end{aligned}
$$

The addend in (41) that does not contain cosine has already been calculated in the previous paragraph (see (32)). Now we should calculate the oscillating addends.

It is easy to calculate that for $b-a \geq 2 \cdot 10^{-7} \mathrm{~cm}$ and the energies that have order of magnitude of Fermi energy arguments of cosines in (41) are much bigger than unity. It means that cosines in (41) are quickly oscillating functions of energy. So an essential contribution into integral (41) are made only by addends with $s=s^{\prime}$, that contain difference of cosine, arguments:

$$
\varphi_{s}(E) \equiv s \frac{2(b-a)}{\hbar} \sqrt{2 m_{0}}(\sqrt{E+\hbar \omega}-\sqrt{E})
$$

This happens because these addends oscillations are the slowest and, consequently, their contribution into integral (41) dominates.

If we leave in (41) only addends of the type of (42), we obtain

$$
\sigma(\omega)=\frac{32 e^{2}(b-a)^{2}}{3 \pi^{3} \hbar^{4} \omega^{3} V_{b}} \int_{0}^{\infty} d E \cdot E^{\frac{3}{2}}(E+\hbar \omega)^{\frac{1}{2}} f(E)(1-f(E+\hbar \omega))\left(1+2 \sum_{s=1}^{\infty} \cos \varphi_{s}^{\prime}(E)\right)
$$

Now we can see that the problem is mathematically analogous with the problem of de Gaaz-van Alfen oscillations (see [4]). So we use analogous approximations, but first, we use the equality

$$
f(E)(1-f(E+\hbar \omega))=\left(1-e^{-\hbar \omega / \theta}\right)^{-1}(f(E)-f(E+\hbar \omega))
$$

In (44) $\theta$ is the temperature (in energetic units). Equality (44) allows us to limit calculation of integral (43) that contains only $f(E)$. An analogous integral that contains $f(E+\hbar \omega)$ can be obtained after replacing Fermi energy in previous integral.

Now, let us expand function $\varphi_{s}(E)$ into series in the vicinity of Fermi energy:

$$
\varphi_{s}(E) \approx \varphi_{s}\left(E_{f}\right)+\varphi_{s}^{\prime}\left(E_{f}\right)\left(E-E_{f}\right)
$$

Now integral from (43) could be written in the for

$$
\begin{aligned}
& \int_{0}^{\infty} d E \cdot E^{\frac{3}{2}}(E+\hbar \omega)^{\frac{1}{2}} f(E) \cos \varphi_{s}(E) \\
& \approx \operatorname{Re}\left(\mu^{\frac{3}{2}}(\mu+\hbar \omega)^{\frac{1}{2}} \exp \left(i \varphi_{s}\left(E_{f}\right)\right) \int_{0}^{\infty} d E f(E) \exp \left(i \varphi_{s}^{\prime}\left(E_{f}\right)\left(E-E_{f}\right)\right)\right) .
\end{aligned}
$$

In (46) we put factor before exponent into the integral (for $E=E_{f}$ ). If oscillations amplitude needs to be calculated more precisely, this function can be expanded in a series analogously to (45).

Now, let us take into account the following: 


$$
\begin{aligned}
\int_{0}^{\infty} d E f(E) \exp \left(i \varphi_{s}^{\prime}\left(E_{f}\right)\left(E-E_{f}\right)\right) & =\theta \int_{-\frac{E_{f}}{\theta}}^{\infty} \frac{d y \cdot \exp \left(i \varphi_{s}\left(E_{f}\right) y\right)}{e^{y}+1} \\
& \approx \theta \int_{-\infty}^{\infty} \frac{d y \cdot \exp \left(i \varphi_{s}\left(E_{f}\right) y\right)}{e^{y}+1}=-\frac{i \pi \theta}{\sinh \left(\pi \varphi_{s}^{\prime}\left(E_{f}\right) \theta\right)}
\end{aligned}
$$

Now, we have all the necessary equations to obtain the final expression for a high-frequency conductivity of metal shell considering discreteness of electronic spectrum:

$$
\sigma_{1}(\omega)=\sigma_{1}^{0}(\omega)+\frac{64 e^{2}(b-a)^{2}}{3 \pi^{2} \hbar^{4} \omega^{3} V_{b}} \theta\left(1-e^{-\frac{\hbar \omega}{\theta}}\right)^{-1}\left(\Phi\left(E_{f}\right)-\Phi\left(E_{f}-\hbar \omega\right)\right)
$$

where the function $\Phi\left(\mathrm{E}_{f}\right)$

$$
\Phi\left(E_{f}\right)=E_{f}^{\frac{3}{2}}\left(E_{f}+\hbar \omega\right)^{\frac{1}{2}} \sum_{s=1}^{\infty} \frac{\sin \varphi_{s}\left(E_{f}\right)}{\sinh \left(\pi \theta \varphi_{s}^{\prime}\left(E_{f}\right)\right)}
$$

and $\sigma_{1}^{0}$ is optical conductivity of a nanoshell without considering discreteness of electronic spectrum (see (32)).

In (48) $V_{b}$ is absorption volume of the nanoshell:

$$
V_{b}=\frac{4 \pi}{3}\left(b^{3}-a^{3}\right)
$$

For thin nanoshells

$$
V_{b} \approx 4 \pi a^{2}(b-a)
$$

We can use the fact that $\hbar \omega<E_{f}$ and expand into series $\varphi_{s}\left(E_{f}\right)$ by $\hbar \omega$ (see $\left.(42)\right)$, we obtain

$$
\varphi_{s} \approx s(b-a)\left(\frac{2 m_{0}}{E_{f}}\right)^{\frac{1}{2}} \omega=s \cdot 2(b-a) \frac{\omega}{v_{f}}=s \tau \omega
$$

in (50) $v_{f}$ is the Fermi speed, $\tau$ is the time of electron flight from inner surface of shell to external and back $\left(\tau=2(b-a) / v_{f}\right)$. We can see that when photon energy $\hbar \omega$ is negligible a comparing to Fermi energy, oscillations possess completely classical nature.

In our approximation, the inequality $\hbar \omega<E_{f}$ is essential because for energies $E^{\prime}=E-\hbar \omega$ inequality (7) must be fulfilled. But, as is mentioned in Appendix, for thin shells $((b-a) / b \ll 1)$ the solution could be found without using inequality (7).

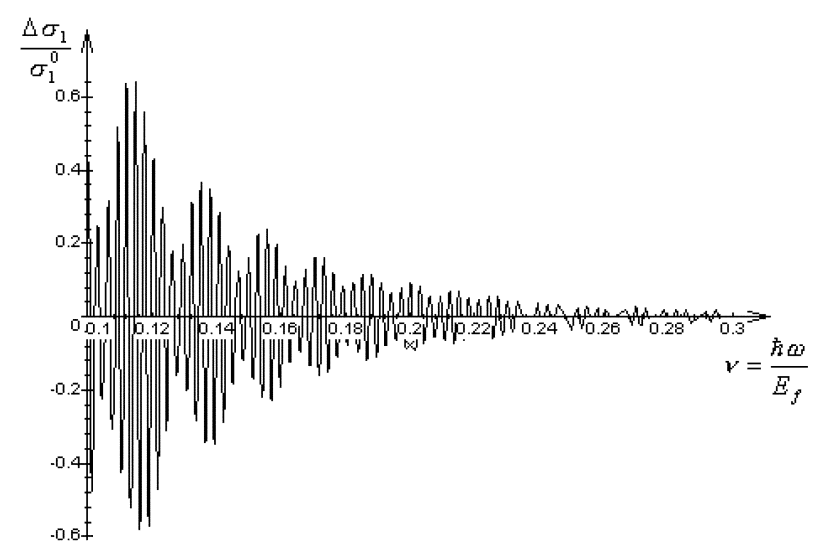

Fig. 2. Relative oscillative addend (for 2 addends in (49)) as a function of incident photon energy - Fermi energy ratio. Graph is given for Fermi energy of nanoshell metal $E_{f}=5.53 \mathrm{eV}$ (Fermi energy of gold), internal radius of the nanoshell $a=40 \mathrm{~nm}$, nanoshell thickness $b-a=100 \mathrm{~nm}$. Interval of $\nu$ is chosen so that $\Delta \sigma_{1}$ is small relative to $\sigma_{1}^{0}$ and $\nu=\hbar \omega / E_{f} \ll 1$.

Now we give graphical representation of the result. We suppose that Fermi energy of nanoshell metal $E_{f}=5.53 \mathrm{eV}$ (Fermi energy of gold), temperature $T=$ $300 \mathrm{~K}$, internal radius of the nanoshell $a=40 \mathrm{~nm}$, nanoshell thickness $b-a=100 \mathrm{~nm}$. Our calculations show that for $T=300 \mathrm{~K}$ factor $\left(1-e^{-\frac{\hbar \omega}{\theta}}\right)^{-1}$ is negligible for practical values of photon energy (it could be replaced with 1). The graphs show that we can leave only 2 addends in sum (49). The dependence of relative oscillating addend $\Delta \sigma_{1} / \sigma_{1}^{0}$, where $\Delta \sigma_{1}$ is the second addend in (48), from photon energy Fermi energy ratio $\nu$ for 2 addends in (49) is given in Fig. 2. An analogous dependence for $b-a=50 \mathrm{~nm}$ and 5 addends in (49) is shown in Fig. 3 (for $b-a=50 \mathrm{~nm}$ we can leave 5 addends in sum (49)). 


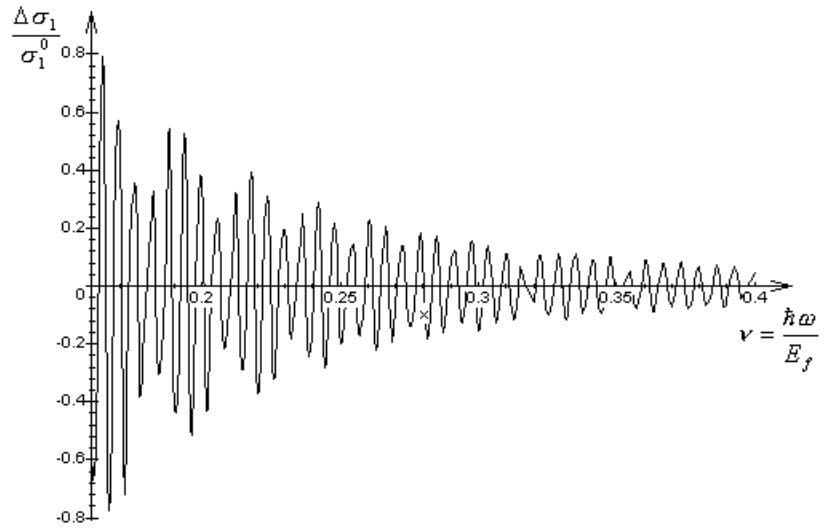

Fig. 3. Relative oscillation addend (for 5 addends in (49)) as a function of incident photon energy - Fermi energy ratio. Graph is given for Fermi energy of nanoshell metal $E_{f}=5.53 \mathrm{eV}$ (Fermi energy of gold), internal radius of the nanoshell $a=40 \mathrm{~nm}$, nanoshell thickness $b-a=50 \mathrm{~nm}$. Interval of $\nu$ is chosen so that $\Delta \sigma_{1}$ is small relative to $\sigma_{1}^{0}$ and $\nu=\hbar \omega / E_{f} \ll 1$.

\section{RESULTS AND REMARKS}

In this work the expression for optical conductivity of spherical metal nanoshell is obtained. Quantization of electron energy in nanoshells is shown to lead to the appearance of an oscillating dependence of optical conductivity from the external electromagnetic field frequency. Oscillations also depend on thickness of metal shell and Fermi energy. An explicit expression of oscillating addends for optical conductivity is obtained.

To perform summation over discrete quantum indices we used the Poisson summation formula and, as electronic spectrum of nanoshells possess quasi-one-dimensional nature, the problem of the investigation of oscillations of optical conductivity becomes mathematically analogous with that of the investigation of de Gaaz-van Alfen oscillations.

In the calculus of optical conductivity according to (41) we left only those addends for which $s=s^{\prime}$ (because cosines oscillate quickly). But situations when $s \sqrt{E_{f}+\hbar \omega} \approx s^{\prime} E_{f}$, so such $s$ and $s^{\prime}$ cannot be neglected, are, in principle, possible.

And finally, we point out that the electric field $E_{0}$ that figures in this work is the one inside the conductor. It can differ from that outside (see, for example, [1]).

\section{APPENDIX}

For thin shells $((b-a) / b \ll 1)$ equation $(3)$ can be solved in another way, if we neglect the dependence of coefficients from (3) from the coordinate the interval $a \leq r \leq b$. Instead of (3) we can write

$$
R^{\prime \prime}+\frac{2}{b} R^{\prime}+\left(k_{n l}^{2}-\frac{l(l+1)}{b^{2}}\right) R=0 .
$$

The solution of (A1) can be written in the form

$$
R=C_{1} e^{\lambda_{1} r}+C_{2} e^{\lambda_{2} r},
$$

where

$$
\lambda_{1,2}=-\frac{1}{b} \pm i \eta \equiv-\frac{1}{b} \pm i \sqrt{k_{n l}^{2}-\frac{l^{2}+l+1}{b^{2}}} .
$$

After taking into consideration (A3) expression (A2) can be transformed:

$$
R=\text { const } \cdot e^{-\frac{r}{b}} \sin \left(\eta r+\varphi_{0}\right) .
$$

Condition $R(a)=R(b)=0$ gives

$$
\eta=\frac{\pi n}{b-a}, \quad \varphi_{0}=-\frac{\pi n a}{b-a}
$$

From the definition of $\eta$ using (A3) we obtain

$$
\eta^{2}=k_{n l}^{2}-\frac{l^{2}+l+1}{b^{2}}
$$

So

$$
k_{n l}^{2}=\eta^{2}+\frac{l^{2}+l+1}{b^{2}}=\left(\frac{\pi n}{b-a}\right)^{2}+\frac{l^{2}+l+1}{b^{2}}
$$

and, consequently, eigennumbers of energy equal

$$
E_{n l}=\frac{\hbar^{2}}{2 m_{0}} k_{n l}^{2}=\frac{\hbar^{2}}{2 m_{0}}\left(\left(\frac{\pi n}{b-a}\right)^{2}+\frac{l^{2}+l+1}{b^{2}}\right)
$$

This result is similar to the result obtained in [5].
[1] R. D. Fedorovich, A. G. Naumovets, P. M. Tomchuk, Phys. Rep. 328, 74 (2000).

[2] P. M. Tomchuk, B. P. Tomchuk, Zh. Eksp. Teor. Fiz. 112 661 (1997).

[3] R. D. Averitt, S. I. Westcott, N. J. Halas, Phys. Rev. B
58, 203 (1998).

[4] A. A. Abrikosov, Osnovy teorii metallov [Basis of metal theoryl (Nauka, Moscow, 1987).

[5] V. A. Arutyunyan, Fiz. Tekhn. Poluprovodn. 36, 401 (2002). 


\title{
ОПТИЧНА ПРОВІДНІСТЬ МЕТАЛЕВИХ НАНООБОЛОНОК
}

\author{
П. М. Томчук ${ }^{1}$, В. В. Куліш ${ }^{2}$ \\ ${ }^{1}$ Інститут фізики, відділ теоретичної фізики, просп. Науки, 46, Київ, 03028, Украӥна, \\ ${ }^{2}$ Фізико-технічний інститут, НТУУ “КПІ", кафедра прикладної фізики, \\ просп. Перемоги, 37, Київ, 03056, Украӥна
}

Отримано вираз для оптичної провідности сферичної металевої оболонки як функції внутрішнього й зовнішнього радіуса нанооболонки й відношення енергіï фотона до енергії Фермі. Показано, що квантування енергїі електрона в нанооболонках зумовлює появу осциляційної залежности оптичної провідности від частоти світла. Отримано явний вигляд осциляційних складових в оптичній провідності. 